\title{
Embedding Media with Microscopic-scale Electrical Conductivity
}

\author{
G.M. Brown, D.W. Abmayr and J.E. Cox
}

Exxon Mobil Chemical Company, Baytown Polymers Center, 5200 Bayway Drive, Baytown, Texas, $77520-2101$

Conventional, non-conductive embedding media, such as epoxy or acrylic resins, are not useful for embedding insulating particles prior to imaging and/or elemental analysis by SEM. The problem is that the cross-sectioned sample builds up electrostatic charge during analysis in the electron microscope unless it is coated with a conductive film. Electrically conductive embedding media have been developed by several manufacturers for embedment of materials prior to polishing and analysis by SEM [1]. These conductive embedding media typically consist of an epoxy matrix containing dispersed metal filler particles. Such commercially available resins exhibit only macroscopic conductivity and are inadequate where a non-charging matrix is desirable for the microscopic analysis of particles. Metal or carbon coating is thus required prior to analysis. FE-SEM analyses of selected conductive epoxies show pronounced electrostatic charging of the insulating epoxy matrix in which islands of the electrically conductive filler particles (i.e. silver, nickel, and copper) are interspersed [2]. The successful analysis of insulating materials, such as aerogel catalyst support particles, in these embedding media is futile because of charge build-up. Flandin et al. cited examples of conductive epoxy thermosets containing $<1$ vol\% carbon black (CB) in which the "macroscopic" electrical conductivity was related to the microstructure of the product [3]. Such conductive embedding materials may exhibit adequate macroscopic conductivity while lacking the submicron-scale microscopic conductivity needed for our applications.

We have developed two types of embedding media that are electrically conductive on a microscopic scale and are useful in the sample preparation of insulating particles for analysis by electron microscopy and EDS. First, an epoxy-based composite was prepared by manually blending N-110 carbon black into an epoxy resin and accelerator mixture [4]. A mortar and pestle was used to produce a uniform composite containing $33 \mathrm{wt} \% \mathrm{CB}$. A second class of microscopically conductive composites utilized functionalized polyethylene copolymers and $\mathrm{CB}$ filler. Ethylene vinylacetate copolymer (EVA) and ethylene methacrylate copolymer (EMA) were physically compounded with $\mathrm{N}-110$ carbon black to produce EVA/CB and EMA/CB composites containing $50 \mathrm{wt} \% \mathrm{CB}$.

Sample embedment is straightforward for each method. Silica aerogels were gently mixed by hand into the uncured epoxy/CB preparation, allowing time for epoxy to infiltrate into the porous structure of aerogel particles prior to heat curing. Ultrathin sections were cut on an ultramicrotome for analysis by TEM/STEM and EDS; alternatively, the bulk face remaining after ultramicrotomy was also available for analysis by SEM and EDS. The remaining cross-sectional face of the bulk sample was also available for analysis by FE-SEM and EDS; FIG. 1. In the EVA/CB or EMA/CB method, silica aerogel particles were sandwiched between two films of the elastomeric CB-filled composite and gently compression molded in a heated hydraulic press. Care was taken not to crush the aerogel particles. This preparation yielded cross-sections of silica aerogel particles in which the EMA or EVA did not infiltrate into the porous structure of the particles. The nascent internal morphologies of the catalyst particles were thus examined by FE-SEM and EDS without the complicating presence of a polymer matrix within the particles; FIG. 2 and 3. 
The advantage of these CB-loaded, electrically conductive composite is that the insulating particles, such as silica aerogels, embedded in the conductive composite exhibit little or no electrostatic charging under the electron beam. Likewise, the composite surrounding the particles does not charge. The absence of electrostatic charging of insulating particles embedded in microscopically conductive CB-filled composites provides several advantages over macroscopically conductive media containing metal filler particles: (1) excellent image contrast in secondary, backscattered and transmitted electron imaging modes is achieved for insulating particles embedded in the conductive carbonaceous composites; (2) the CB-based composites are devoid of metal fillers whose X-ray lines may interfere with $\mathrm{x}$-ray lines of the catalyst metals supported on silica aerogels; (3) excellent elemental contrast is seen between silica particles and the carbonaceous matrix of the composite in EDS spectra and maps; and (4) electrically conductive matrices minimize the build-up of electrostatic charge on insulating catalyst particles, thus providing opportunities for high quality imaging and spectroscopic analysis of embedded particles. [5]

\section{References}

[1] See vendors of metallography equipment and suppliers.

[2] One of two electrically conductive embedding media tested is a commercially available, silverloaded epoxy.

[3] L. Flandin, et al., Materiaux \& Techniques, 7-8, (2000), 51.

[4] Epo-Fix embedding medium, from Electron Microscopy Sciences (www.emsdiasum.com)

[5] Thanks to Dr. Andy Tsou for compounding the functionalized polyethylene/CB composites.
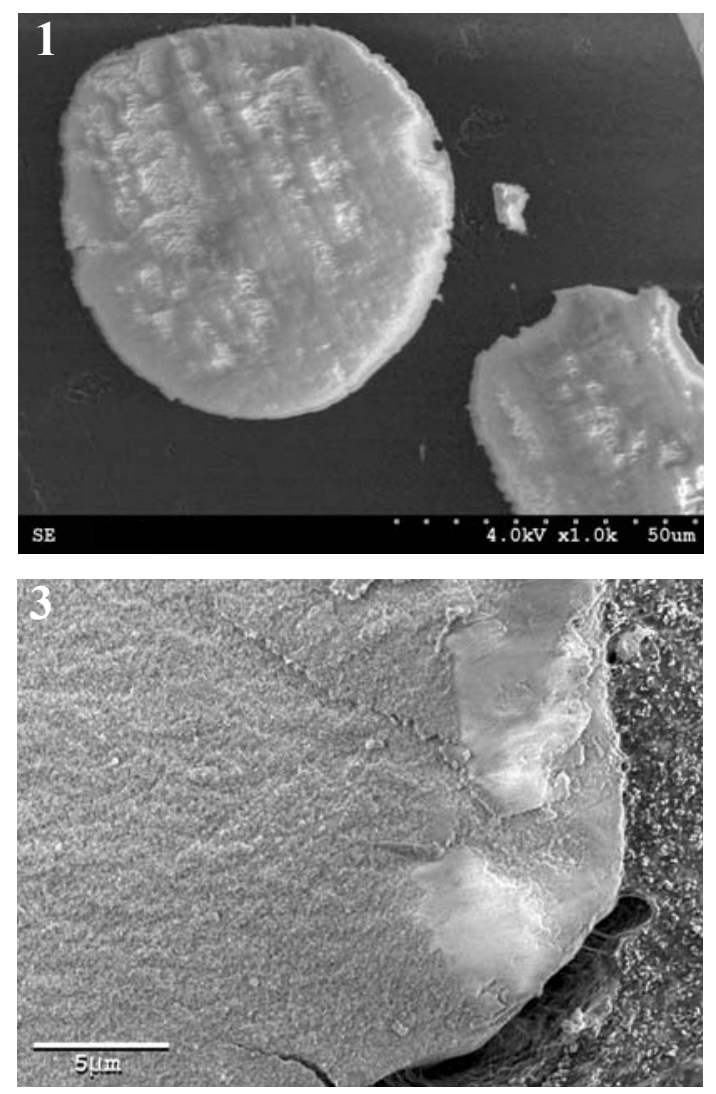

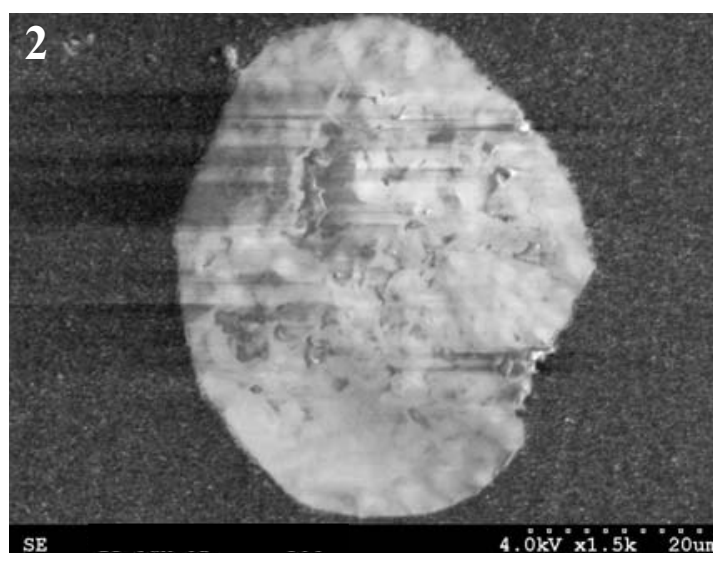

FE-SEM images of silica support particles (aerogels) embedded in conductive media. FIG. 1. Particles embedded in an epoxy/CB composite show little charging in the matrix and submicron-scale electrical conductivity in the matrix. FIG. 2. A silica-supported catalyst particle is shown embedded in an EVA/CB composite containing $33 \mathrm{wt} \% \mathrm{CB}$. Aggregates of $\mathrm{CB}$ may be seen as lighter specks in the EVA matrix. The insulating particle charges only very slightly (black streaks) at $4 \mathrm{kV}$; the CBcontaining matrix did not charge even at $15 \mathrm{kV}$. FIG. 3. A portion of a silica catalyst support particle is shown embedded in an EVA/CB (33 wt\% CB). At 5,000x and 1 $\mathrm{kV}$, the variably porous structure of the silica is readily imaged. 